\title{
Improved annotation and quantification of metabolites in rice (Oryza sativa L.) seeds using two-dimensional gas chromatography- time-of-flight mass spectrometry
}

Seung-A. Baek ${ }^{1,4^{\dagger}}$, So Yeon Kim ${ }^{1 \dagger}$, Young Jin Park ${ }^{1}$, Tae Jin Kim ${ }^{1,5}$, Sun-Hyung Lim², Sang Un Park ${ }^{3^{*}}$ and Jae Kwang Kim ${ }^{*}$ (I)

\begin{abstract}
Two-dimensional gas chromatography coupled to time-of-flight mass spectrometry (GC $\times$ GC-TOFMS) is a powerful tool for identification of compounds in complex samples. Herein, we compared the GC × GC-TOFMS and GCTOFMS systems for polar metabolite profiling of rice seeds. Forty-seven and thirty-six metabolites were detected in a Korean rice cultivar, Dongjin, using GC $\times$ GC-TOFMS and GC-TOFMS, respectively. The limits of detection of shikimic, p-coumaric, and sinapinic acids were 30.0-, 1.6-, and 2.5-times lower, respectively, with GC × GC-TOFMS than with GC-TOFMS. The overlapped peaks of glycerol and phosphoric acid in GC-TOFMS were separated in GC $\times$ GC-TOFMS. Polar metabolite profiling of two white and six red rice cultivars was performed using GC $\times$ GC-TOFMS and the obtained data were subjected to principal component analysis. Remarkably, principal component 1 separated Heugdaegu from other cultivars, indicating that Heugdaegu has high levels of caffeic, sinapinic, and vanillic acids. Findings from this work may aid breeding programs aimed at improving the quality of rice seeds.
\end{abstract}

Keywords: GC × GC-TOFMS, GC-TOFMS, Metabolic profiling, Oryza sativa L., Pigmented rice

\section{Introduction}

Polar primary metabolites are essential for vital activities of organisms. In contrast, secondary metabolites are not directly involved in the normal growth, development, and reproduction, but several such metabolites are present in plants for ecological purposes. Because primary metabolites are used as precursors of other primary metabolites or as building blocks for the synthesis of secondary

\footnotetext{
*Correspondence: supark@cnu.ac.kr; kjkpj@inu.ac.kr

${ }^{\dagger}$ Seung-A. Baek and So Yeon Kim contributed equally to this work

${ }^{1}$ Division of Life Sciences and Bio-Resource and Environmental Center,

College of Life Sciences and Bioengineering, Incheon National University,

Incheon 22012, Republic of Korea

${ }^{3}$ Department of Crop Science and Department of Smart Agriculture

Systems, Chungnam National University, 99 Daehak-ro, Yuseong-gu,

Daejeon 34134, Republic of Korea

Full list of author information is available at the end of the article
}

metabolites, study of biosynthetic pathway of metabolites is important for understanding the plant physiology and for designing new plants with enhanced nutritional value. Towards this end, profiling of primary metabolites and analysis of the relationship between the metabolites in various plants has been performed using GC-TOFMS [1-4]. Researchers in the field of metabolomics have been striving to improve the chromatographic resolution and detection sensitivity of all metabolites present in a sample.

Comprehensive two-dimensional gas chromatography $(\mathrm{GC} \times \mathrm{GC})$ is an advanced technique that improves the resolution of one-dimensional gas chromatography (GC). Two columns, with different stationary phases, are connected through a modulator (thermal or valvebased modulator). The eluates from the first column are transferred into the second column and are concentrated 
during the transfer in the modulator [5]. GC $\times \mathrm{GC}$ coupled to quadrupole mass spectrometry (qMS) or time-of-flight mass spectrometry (TOFMS) reduces the probability of peak overlap and increases the number of detected peaks [6-8]. GC $\times$ GC-TOFMS has higher selectivity and greater sensitivity than GC-TOFMS $[5,8$, 9], and has, therefore, been used for metabolic analysis of several plants including brown rice seeds. However, to the best of our knowledge, metabolite profiling of pigmented rice seeds using GC $\times$ GC-TOFMS has not been reported [10-12].

In plant metabolomics, GC $\times$ GC-TOFMS has been applied to metabolite fingerprinting with chemometric tools. After non-targeted metabolic profiling, comparison of data obtained from samples (i.e., control and treated samples, cultivars, or species) with multivariate statistics is performed [13-16]. In the case of rice grains, GC $\times$ GC-TOFMS has been used for non-targeted metabolomic studies. Volatile metabolites in 10 rice varieties were assayed to understand the mechanism of synthesis of aroma compounds [17]. Eight volatile compounds were selected as key markers responsible for the differences between aromatic and non-aromatic rice varieties [18]. Volatiles collected from microbe- and mite-contaminated rice grain were analysed using GC $\times$ GC-TOFMS for assessment of contamination [14]. Non-targeted profiling of polar metabolites in five brown cultivars was performed using GC $\times$ GC-TOFMS in combination with GC-TOFMS analysis for investigating the metabolite diversity of rice variants [10]. In addition, non-targeted profiling of polar metabolites in grains of three rice cultivars was performed using GC-TOFMS and the results were compared with those obtained using GC $\times$ GC-TOFMS analysis for assessing the data processing and analysis methods [11].

Non-targeted metabolite profiling has been important in discovering biomarkers and for screening unrevealed metabolites in samples; however, in plant metabolomics the focus has been on the already known metabolites related to pathways including the tricarboxylic acid cycle, glycolysis, and biosynthesis of secondary metabolites. Physiological mechanisms have been analysed by comparing the levels of metabolites in samples that were mutated or exposed to stimuli with those in the respective control samples [19-21]. In this study, we performed targeted metabolic profiling in rice seed using GC $\times$ GC-TOFMS for the first time. Moreover, we compared the GC $\times$ GC-TOFMS platform with GC-TOFMS for analysis of polar metabolites in rice seed. The number of metabolites detected using GC-TOFMS was compared with those detected using GC $\times$ GC-TOFMS. Limit of detection (LOD), limit of quantification (LOQ), accuracy, and precision of standards were measured to compare the performance of GC-TOFMS and GC $\times$ GC-TOFMS. Subsequent to method validation, polar metabolites extracted from two white and six red pigmented rice cultivar seeds were analysed using GC $\times$ GC-TOFMS. The metabolic profiling data were analysed by principal component analysis (PCA) for comparing the metabolites among the eight rice cultivars.

\section{Materials and methods \\ Samples}

Eight varieties of Korean rice seeds were categorized as white and red according to the colour of their pericarp. Two cultivars of white rice (Dongjin, DJ; Heugdaegu, HDG) and six of red rice (Aengmi, AM; Goryeong 8, GR8; Hanyangjo, HYJ; Hongjinju, HJJ; Jakwangdo, JKD; Jeogiinju, JJJ) were used in this study. The seeds were obtained from the Agricultural Genetic Resources Center at the National Academy of Agricultural Science (Suwon, Korea). The seeds were harvested in 2016 and manually hulled and ground to a fine powder with a mixer mill (HR2860, Philips, Amsterdam, The Netherlands) and pestle and mortar. The powdered seeds were stored at $-20^{\circ} \mathrm{C}$ until they were used.

\section{Chemicals}

Methanol (HPLC grade) and chloroform (HPLC grade) were purchased from Daejung Chemical \& Metal (Siheung, Korea) and Burdick \& Jackson (Muskegon, MI, USA), respectively. Water used in the experiments was prepared using the Millipore water purification system (Milli-Q Direct 8; Milford, MA, USA). Adonitol (ribitol; $\geq 99 \%$ ), shikimic acid ( $\geq 99 \%)$, sinapinic acid ( $\geq 98 \%$ ), methoxyamine hydrochloride (MOX; 98\%), pyridine (HPLC grade, $\geq 99.9 \%$ ), and $N$-methyl- $N$-(trimethylsilyl) trifluoroacetamide (MSTFA) were purchased from Sigma (St. Louis, MO, USA). p-Coumaric acid was purchased from MP Biomedicals (Solon, OH, USA).

\section{Extraction of polar metabolites}

Polar metabolites, including amino acids, organic acids, sugars, and phenolic acids, in pigmented rice $(\mathrm{n}=3)$ were extracted following a previously described method [1]. Briefly, $10 \mathrm{mg}$ powder was mixed with $1 \mathrm{~mL}$ of 2.5:1:1 (v/v/v) methanol:chloroform:water containing $60 \mu \mathrm{L}$ adonitol $(200 \mu \mathrm{g} / \mathrm{mL})$ as an internal standard (IS). After shaking at $1200 \mathrm{rpm}$ for $30 \mathrm{~min}$ at $37^{\circ} \mathrm{C}$ (Eppendorf Thermomixer Comfort 5355, Eppendorf, Hamburg, Germany), the mixture was centrifuged at $16,000 \times g$ for 3 min at $4{ }^{\circ} \mathrm{C}$ (MX-307, TOMY, Tokyo, Japan). The upper layer $(800 \mu \mathrm{L})$ was transferred to a new tube and $400 \mu \mathrm{L}$ water was added. The mixture was vortexed and centrifuged at $16,000 \times g$ for $3 \mathrm{~min}$ at $4{ }^{\circ} \mathrm{C}$. The methanol:water phase $(900 \mu \mathrm{L})$ was transferred to a new tube and dried 
completely using a vacuum centrifuge dryer (VS-802F, Visionbionex, Gyeonggi, Korea) and a freeze-dryer (MCFD8512, IlShinBioBase, Gyeonggi, Korea). For derivatisation, $80 \mu \mathrm{L} 2 \% \mathrm{MOX}$ (in pyridine, w/v) was added to the sample and incubated at $30{ }^{\circ} \mathrm{C}$, with shaking at $1200 \mathrm{rpm}$ for $90 \mathrm{~min}$. Thereafter, $80 \mu \mathrm{L}$ MSTFA was added and the mixture was incubated at $37{ }^{\circ} \mathrm{C}$, with shaking at $1200 \mathrm{rpm}$ for $30 \mathrm{~min}$. The sample was moved to a vial for GC-TOFMS and GC $\times$ GC-TOFMS analyses.

\section{GC-TOFMS and GC $\times$ GC-TOFMS conditions}

Agilent 7890A GC (Agilent, Santa Clara, CA, USA) coupled to a Pegasus TOFMS 4D (LECO, St Joseph, MI, USA) was used for the analysis of polar metabolites using GCTOFMS and GC $\times$ GC-TOFMS. An Rtx-5MS column $(0.25 \mathrm{~mm} \times 0.25 \mu \mathrm{m} \times 30 \mathrm{~m}$; Restek, Bellefonte, PA, USA) was used for GC-TOFMS analysis. For GC-TOFMS analysis, the column oven temperature was maintained at $80{ }^{\circ} \mathrm{C}$ for $0.5 \mathrm{~min}$, was increased at $5{ }^{\circ} \mathrm{C} / \mathrm{min}$ to $330{ }^{\circ} \mathrm{C}$ and then maintained at $330^{\circ} \mathrm{C}$ for $5 \mathrm{~min}$. For GC $\times$ GCTOFMS, Rtx-5MS $(0.25 \mathrm{~mm} \times 0.25 \mu \mathrm{m} \times 30 \mathrm{~m}$; Restek $)$ and Rxi-17sil MS $(0.15 \mathrm{~mm} \times 0.15 \mu \mathrm{m} \times 1.2 \mathrm{~m}$; Restek $)$ columns were used as the first and second columns, respectively. The oven conditions for the first column were the same as in GC-TOFMS, whereas the second column oven temperature program was $5{ }^{\circ} \mathrm{C}$ above that of the first column. The two columns were connected through a cryogenic modulator and liquid nitrogen was used as the cryogen. The modulator temperature program was $15{ }^{\circ} \mathrm{C}$ above the second column temperature. The modulation period was set to $4 \mathrm{~s}$, with $0.6 \mathrm{~s}$ hot and $1.4 \mathrm{~s}$ cool pulse duration. Except for the column oven condition, other conditions were the same for both GCTOFMS and GC $\times$ GC-TOFMS analyses. One microlitre of derivatised sample was injected in the split (25:1) mode at $250{ }^{\circ} \mathrm{C}$. Helium was used as the carrier gas at a flow rate of $1.2 \mathrm{~mL} / \mathrm{min}$ in a constant flow mode. The temperature of transfer line and ion source was 260 and $230{ }^{\circ} \mathrm{C}$, respectively. The mass electron energy was set at $-70 \mathrm{eV}$ and the mass range for scanning was in the $45-650 \mathrm{~m} / z$ range. The mass acquisition rate was 10 spectra/sec in GC-TOFMS and 100 spectra/sec in GC $\times$ GC-TOFMS. The qualitative analysis was performed by comparison with the retention time of respective standards and mass spectrum in an in-house library, Wiley9, and NIST14 using the ChromaTOF software (V4.50, LECO). The quantitative estimation was based on peak area ratios relative to the peak area of the IS.

\section{Analytical method validation}

Six different concentrations of shikimic acid $(0.1-5.0 \mu \mathrm{g})$, $p$-coumaric acid $(0.25-12.5 \mu \mathrm{g})$, and sinapinic acid $(0.75-12.5 \mu \mathrm{g})$ standards were analysed within 1 day of preparation to determine linearity, precision, and accuracy $(\mathrm{n}=3)$. Ribitol $(12 \mu \mathrm{g})$ was added as an IS to each standard sample. The linearity was calculated as the square of the correlation coefficient $(r)$ of the calibration curve. LOD and LOQ of shikimic acid, sinapinic acid, and $p$-coumaric acid were estimated as the following expressions: $3 \alpha / \mathrm{S}$ (LOD) and $10 \alpha / \mathrm{S}$ (LOQ), where $\alpha$ is the standard deviation $(n=7)$ and $S$ is the slope of the calibration curve. The precision and accuracy were defined as the relative standard deviation (RSD) and recovery, respectively. RSD was calculated using the following equation: $(\alpha / \mu) \times 100$, where $\alpha$ is the standard deviation and $\mu$ is the mean $(n=3)$. Recovery was estimated as $\left(\mathrm{SS}_{\mathrm{C}} / \mathrm{SS}_{\mathrm{A}}\right) \times 100$, where $\mathrm{SS}_{\mathrm{C}}$ is the amount of spiked standard calculated using a calibration curve and $\mathrm{SS}_{\mathrm{A}}$ is the amount of spiked standard.

\section{Multivariate statistical analysis}

Polar metabolite analysis was performed in triplicate. PCA was performed using SIMCA-P (version 13.0; Umetrics, Umeå, Sweden). The data were normalised with unit variance scaling.

\section{Results and discussion \\ Metabolic profiling of DJ using GC-TOFMS and GC $\times$ GC-TOFMS}

To compare the GC-TOFMS and GC $\times$ GC-TOFMS systems, polar metabolite profiling of the same derivate sample (DJ) was performed using the both the instruments. A total 38 polar metabolites were detected in DJ by GC-TOFMS analysis. Twenty one amino acids and amino acid derivatives, six organic acids, seven sugars, phosphoric acid, ferulic acid, $p$-hydroxybenzoic acid and glycerol were identified (Fig. 1A). On the contrary, a total of 47 polar metabolites were detected by $\mathrm{GC} \times \mathrm{GC}$ TOFMS analysis (Fig. 1B). Twenty-two amino acids and amino acid derivatives, eight organic acids, seven phenolic acids, eight sugars, phosphoric acid, and glycerol were identified. Cysteine, methionine, pyruvic acid, shikimic acid, caffeic acid, salicylic acid, sinapinic acid, vanillic acid, $p$-coumaric acid, $p$-hydroxybenzoic acid, and glyceric acid were only detected in the GC $\times$ GCTOFMS analysis (Fig. 1; Additional file 1: Table S1).

\section{Comparison of the sensitivities of GC-TOFMS and GC $\times$ GC-TOFMS}

Shikimic acid is a precursor of aromatic amino acids (phenylalanine and tyrosine) and phenylpropanoid pathway starts with phenylalanine. Flavonoids, monolignols, phenolic acids, stilbenes, and coumarins are derived from phenylalanine through successive consecutive enzymatic reactions in most plants [22]. Kim et al. [1] reported a positive relationship between all phenolic and shikimic 


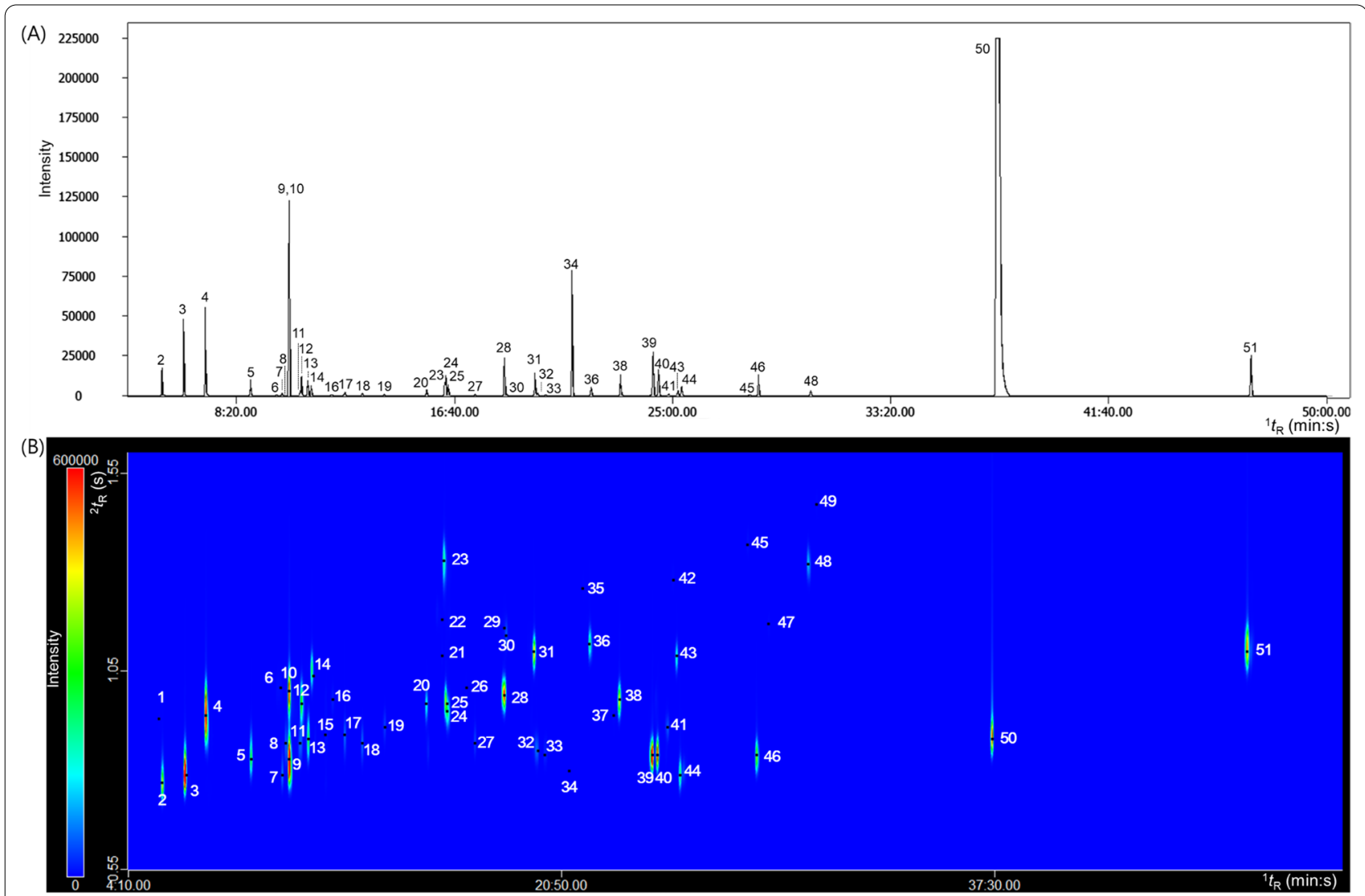

Fig. 1 GC-TOFMS (A) and GC $\times$ GC-TOFMS (B) analytical ion chromatogram (AIC) of polar metabolites extracted from Dongjin. Peak: 1, Pyruvic acid; 2, Lactic acid; 3, Alanine; 4, Oxalic acid; 5, Valine; 6, Serine-1; 7, Ethanolamine; 8, Leucine; 9, Glycerol; 10, Phosphoric acid; 11 , Isoleucine; 12, Proline; 13, Glycine; 14, Succinic acid;15, Glyceric acid; 16, Fumaric acid; 17, Serine-2; 18, Threonine; 19, $\beta$-Alanine; 20, Malic acid; 21, Salicylic acid; 22, Methionine; 23, Pyroglutamic acid; 24, Aspartic acid; 25, 4-Aminobutytic acid; 26, Cysteine; 27, Threonic acid; 28, Glutamic acid; 29, p-Hydroxybenzoic acid; 30, Phenylalanine; 31, Asparagine; 32, Xylose-1; 33, Xylose-2; 34, Ribitol (internal standard); 35, Vanillic acid; 36, Glutamine; 37, Shikimic acid; 38, Citric acid; 39, Fructose-1; 40, Fructose-2; 41, Lysine; 42, p-Coumaric acid; 43, Tyrosine; 44, Mannitol; 45, Ferulic acid; 46, Inositol; 47, Caffeic acid; 48, Tryptophan; 49, Sinapinic acid; 50, Sucrose; 51, Raffinose

acids in black rice cultivars. Several studies have reported that red rice seeds contain more phytochemicals, such as flavone (apigenin) and phenolics, than white rice [23-25]. Therefore, to validate the method, a representative precursor (shikimic acid) and phenolic acid ( $p$-coumaric acid and sinapinic acid) were selected among 10 metabolites that were only detected by GC $\times$ GC-TOFMS analysis. The calibration curves of shikimic acid, $p$-coumaric acid, and sinapinic acid were produced using GC-TOFMS and GC $\times$ GC-TOFMS to compare the LOD and LOQ. The means of three-point data for calculating the ratio relative to the peak area of the IS were used as calibration curves. All the calibration curves showed linearity with a correlation coefficient above 0.99 (Table 1). The LOD of shikimic acid, $p$-coumaric acid, and sinapinic acid was 9.3, 90.2, and $119.6 \mathrm{ng}$ and the LOQ was 30.9, 300.6, and $398.6 \mathrm{ng}$, respectively, in the GC-TOFMS analysis. In GC $\times$ GC-TOFMS, the LOD of shikimic acid, $p$-coumaric acid, and sinapinic acid was $0.3,55.9$, and $47.2 \mathrm{ng}$,
Table 1 Linearity $\left(r^{2}\right)$, limit of detection (LOD), and limit of quantification (LOQ) of shikimic acid, p-coumaric acid, and sinapinic acid analysed using GC-TOFMS and GC $\times$ GC-TOFMS

\begin{tabular}{|c|c|c|c|c|}
\hline Metabolite & Calibration curve & Linearity & LOD (ng) & LOQ (ng) \\
\hline \multicolumn{5}{|l|}{ GC-TOFMS } \\
\hline Shikimic acid & $\begin{array}{l}y=0.0529 x- \\
0.0054\end{array}$ & 0.9963 & 9.26 & 30.88 \\
\hline p-Coumaric acid & $\begin{array}{l}y=0.0080 x- \\
0.0083\end{array}$ & 0.9910 & 90.18 & 300.60 \\
\hline Sinapinic acid & $\begin{array}{l}y=0.0035 x- \\
0.0025\end{array}$ & 0.9920 & 119.58 & 398.59 \\
\hline \multicolumn{5}{|l|}{ GC $\times$ GC-TOFMS } \\
\hline Shikimic acid & $\begin{array}{l}y=0.1486 x+ \\
0.0004\end{array}$ & 0.9978 & 0.31 & 1.03 \\
\hline$p$-Coumaric acid & $\begin{array}{l}y=0.0221 x- \\
0.0022\end{array}$ & 0.9987 & 55.87 & 186.25 \\
\hline Sinapinic acid & $\begin{array}{l}y=0.0176 x- \\
0.0078\end{array}$ & 0.9966 & 47.22 & 157.41 \\
\hline
\end{tabular}


and the LOQ was 1.0, 186.3, and 157.4 ng, respectively. The LOD and LOQ of shikimic acid, sinapinic acid, and $p$-coumaric acid in the GC $\times$ GC-TOFMS analysis were 30.0-, 1.6-, and 2.5-times lower than those of in GCTOFMS, respectively. The analytes eluting from the first column were trapped in the thermal modulator by cold jet flow and then re-injected by hot jet flow into the second column. Because in this step, the eluates are concentrated, it improves the sensitivity of GC $\times$ GC [5]. GC $\times$ GC-TOFMS analyse significantly reduces the matrix effect by separation of second column. In this study, the LOD and LOQ of GC $\times$ GC-TOFMS were better than those of GC-TOFMS.

To determine the precision and accuracy, three different concentrations within each calibration curve range were measured (Table 2). The precision (\%RSD) for shikimic acid, $p$-coumaric acid, and sinapinic acid was lower than $12.9 \%$ in both GC-TOFMS and GC $\times$ GC-TOFMS analyses. The accuracy (\%Recovery) ranged from 85.1 to $114.6 \%$ for the GC-TOFMS and GC $\times$ GC-TOFMS analyses. The precision of GC-TOFMS and GC $\times$ GCTOFMS for the metabolites was lower than $15 \%$. The accuracy (\% Recovery) of GC-TOFMS and GC $\times$ GCTOFMS for the metabolites ranged from 85 to $115 \%$ and was in agreement with the International Guidelines (FDA Guidance) [26]. Thus, the GC-TOFMS and GC $\times$ GC-TOFMS systems were found to be valid tools for polar metabolite analysis, but GC $\times$ GC-TOFMS could detect lower levels of metabolites when compared with GC-TOFMS.

\section{Improved separation ability in GC x GC-TOFMS}

GC $\times$ GC-TOFMS showed a better peak separation ability than GC-TOFMS. The two columns with different properties (mid-polar and non-polar) lead to a better separation of co-eluted analytes from the first column
$[6,27,28]$. In this study, the peaks of glycerol and phosphoric acid overlapped in GC-TOFMS but could be separated and quantified using different fragment ions $(\mathrm{m} / \mathrm{z})$ (Fig. 2A-C). However, the mass spectrum of glycerol was affected by that of phosphoric acid. The unique fragment ion, with a comparatively higher intensity, was selected as the quantitative fragment ion for each metabolite (Fig. 2F, G). The $m / z 103$ and 299 were chosen as quantitative fragment ions of glycerol and phosphoric acid, respectively. However, $m / z 103$ fragment ion was detected in phosphoric acid with $0.8 \%$ relative abundance. The overlapped fragment ion for two metabolites could affect the area of the glycerol peak. Winnike et al. [6] reported that the peak area ratio of each metabolite $\left(R_{P A}\right)$ was expected to be consistent. $\mathrm{R}_{\mathrm{PA}}$ was the calculated peak area of metabolite obtained using the GC-TOFMS analysis divided by that obtained using the GC $\times$ GC-TOFMS analysis. $\mathrm{R}_{\mathrm{PA}}$ of leucine, phosphoric acid, and isoleucine was $0.07,0.07$, and 0.06 , respectively. However, $R_{P A}$ of glycerol was 0.21 . The peak area of glycerol was overmeasured with $\mathrm{m} / \mathrm{z} 103$ fragment ion of phosphoric acid and it might have increased the $\mathrm{R}_{\mathrm{PA}}$.

Moreover, some of the other fragments, including $m / z 73,133,147,175$, and 205 , overlapped. The $m / z$ 133 fragment ion was monitored in both glycerol and phosphoric acid with a relative abundance of $19.9 \%$ and $8.2 \%$, respectively. The prominent fragment ions of glycerol, $\mathrm{m} / \mathrm{z} 147$ and 205, showed a relative abundance of $3.9 \%$ and $1.9 \%$ in phosphoric acid, respectively. This suggests that the user has to finally sort out the fragment ions from each metabolite in an overlapped mass spectrum. In GC $\times$ GC-TOFMS, the glycerol peak also overlapped with that of phosphoric acid in the retention time in first dimension but they were separated in the second dimension (Fig. 2D, E). Thus, the two metabolites did not affect the mass spectrum

Table 2 Precision (\%RSD) and accuracy (\%Recovery) for determination of shikimic acid, $p$-coumaric acid, and sinapinic acid using GC-TOFMS and GC $\times$ GC-TOFMS

\begin{tabular}{|c|c|c|c|c|c|c|}
\hline \multirow[t]{2}{*}{ Metabolite } & \multicolumn{3}{|l|}{ GC-TOFMS } & \multicolumn{3}{|c|}{ GC $\times$ GC-TOFMS } \\
\hline & Content $(\mu \mathrm{g})$ & Precision (\%RSD) & Accuracy (\%Recovery) & Content $(\mu \mathrm{g})$ & Precision (\%RSD) & Accuracy (\%Recovery) \\
\hline \multirow[t]{3}{*}{ Shikimic acid } & 0.50 & 1.86 & $85.12 \pm 1.58$ & 0.05 & 7.55 & $89.95 \pm 6.79$ \\
\hline & 2.50 & 1.70 & $106.85 \pm 1.81$ & 0.10 & 11.24 & $96.95 \pm 10.90$ \\
\hline & 5.00 & 12.90 & $108.89 \pm 14.04$ & 1.00 & 8.02 & $98.22 \pm 7.87$ \\
\hline \multirow[t]{3}{*}{ p-Coumaric acid } & 5.00 & 4.17 & $87.13 \pm 3.63$ & 0.50 & 11.61 & $93.48 \pm 10.85$ \\
\hline & 10.00 & 8.11 & $105.41 \pm 8.54$ & 1.00 & 6.06 & $96.31 \pm 5.83$ \\
\hline & 12.50 & 7.85 & $102.49 \pm 8.05$ & 5.00 & 12.59 & $107.04 \pm 13.47$ \\
\hline \multirow[t]{3}{*}{ Sinapinic acid } & 7.50 & 7.55 & $90.67 \pm 6.84$ & 0.75 & 3.53 & $114.57 \pm 4.04$ \\
\hline & 10.00 & 2.68 & $104.66 \pm 2.80$ & 1.00 & 4.33 & $100.61 \pm 4.36$ \\
\hline & 12.50 & 3.98 & $100.91 \pm 4.02$ & 5.00 & 8.35 & $94.12 \pm 7.86$ \\
\hline
\end{tabular}




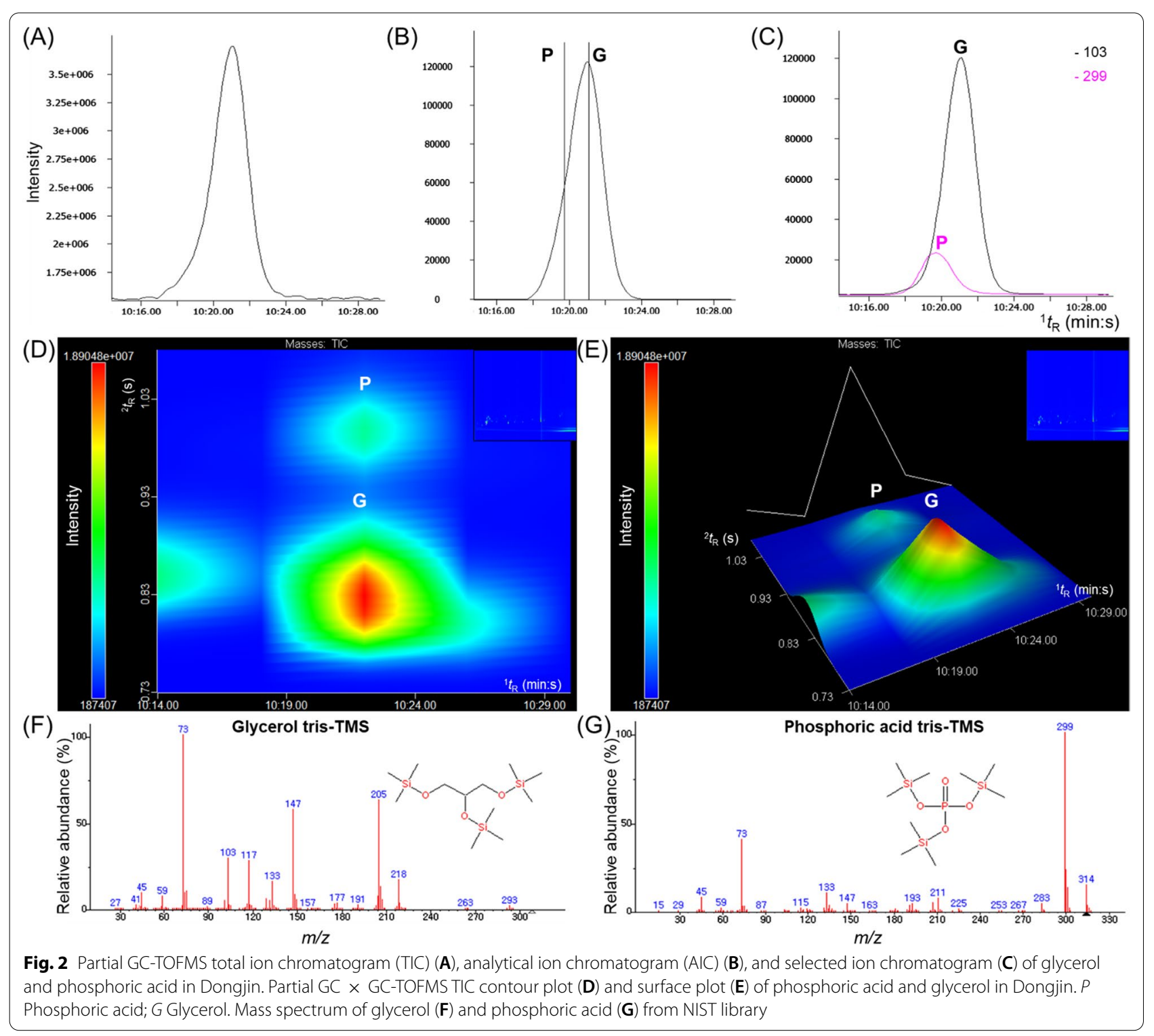

Table 3 Mass spectral similarity of phosphoric acid and glycerol in Dongjin analysed using GC-TOFMS and GC × GC-TOFMS

\begin{tabular}{|c|c|c|c|c|c|}
\hline \multirow[t]{2}{*}{ Peak number } & \multirow[t]{2}{*}{ Library } & \multicolumn{2}{|c|}{ GC-TOFMS } & \multicolumn{2}{|c|}{$\mathrm{GC} \times \mathrm{GC}-\mathrm{TOFMS}$} \\
\hline & & NIST & Wiley9 & NIST & Wiley9 \\
\hline 9 & Glycerol & 860 & 860 & 935 & 927 \\
\hline 10 & Phosphoric acid & 817 & 818 & 873 & 919 \\
\hline
\end{tabular}

and area of each other. In addition, the user could easily determine whether the mass spectrum of a peak is consistent with those of targeted metabolites by referring to the similarity in a library, such as NIST and Wiley (Table 3 ). The separated peak, leading to better spectrum deconvolution, resulted in better selectivity of GC $\times$ GC-TOFMS than that of GC-TOFMS.

\section{Analysis of polar metabolites in two white and six pigmented rice cultivars with GC x GC-TOFMS}

In this study, GC $\times$ GC-TOFMS showed improved sensitivity and peak separation ability compared with GC-TOFMS. Thus, polar metabolite profiling in eight rice cultivars was performed using GC $\times$ GC-TOFMS . As observed for DJ, a total 47 polar metabolites were detected in the seeds of all the cultivars. The obtained data were subjected to PCA to assess the differences in polar metabolite composition among rice seeds (Fig. 3). 

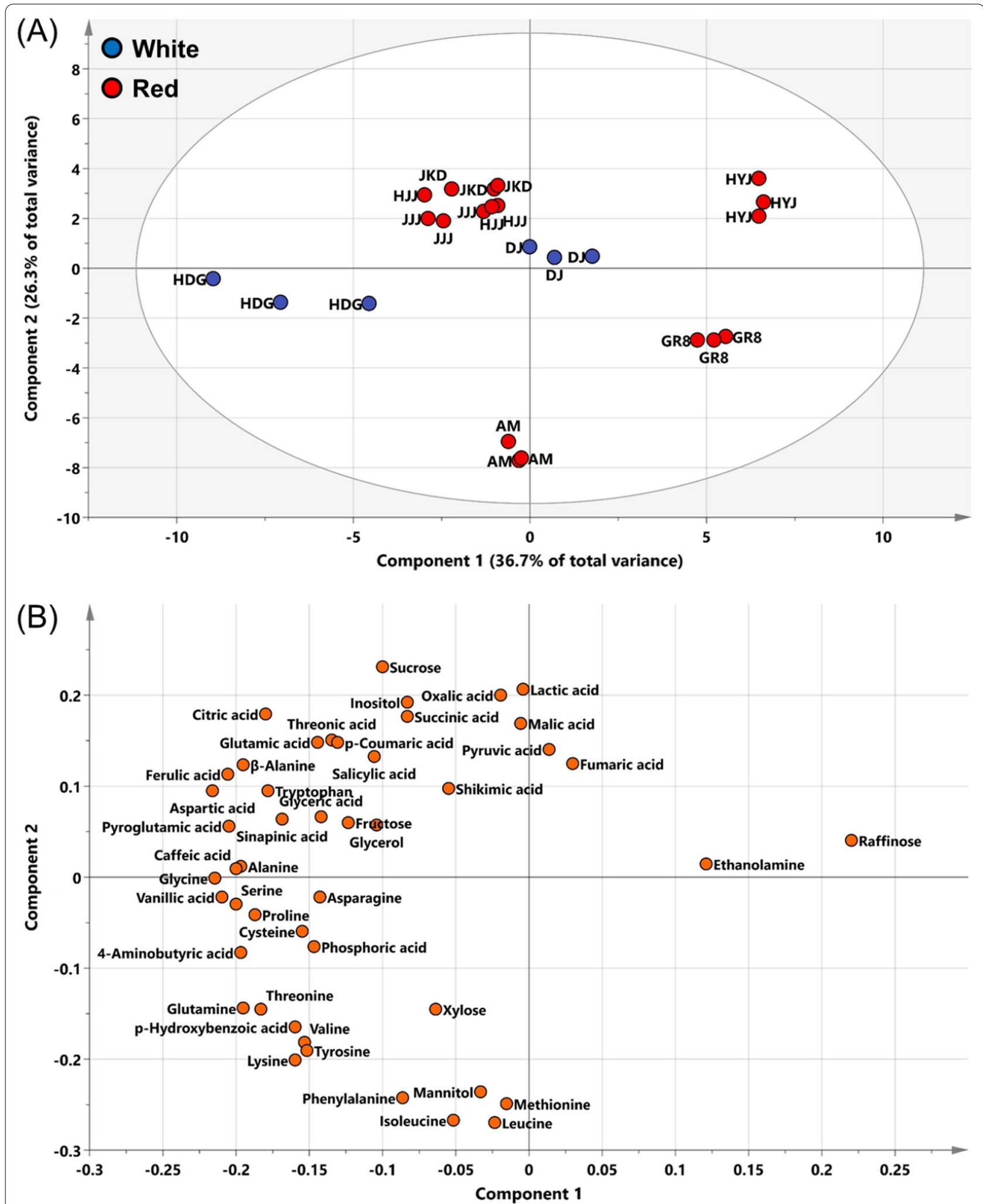

Fig. 3 Score plots (A) and loading plots (B) of principal component analysis (PCA) obtained from metabolic profiling by using GC $\times$ GC-TOFMS analysis. AM Aengmi; GR8 Goryeong 8; HYJ Hanyangjo; HJJ Hongjinju; JKD Jakwangdo; JJ Jeogjinju; DJ Dongjin; HDG Heugdaegu 
PCA is a good tool to obtain an overview of the comprehensive data and has been used in metabolomics studies [29]. In the score plots, each point represents an individual sample, and samples exhibiting similar variances are clustered together. The first two principal components (PCs; PC1 and PC2) had the greatest eigenvalues and captured $63.0 \%$ of the total variance (Fig. 3A). The same cultivar was closely clustered in the score plot but was not clustered with the colour of rice. The white cultivars (blue-coloured spots; DJ and HDG) were not clearly separated with the red cultivars (red-coloured spots; AM, GR8, HYJ, HJJ, JKD, and $\mathrm{JJJ}$ ). However, clear a separation was observed between the HDG cultivar and other cultivars by PC1. Although the HDG has white-coloured pericarp, its hull has black colour. PC2 separated the AM cultivar from other cultivars. Although the AM cultivar belongs to the same species as the cultivated rice, it is referred to as weedy rice in Korea. The corresponding loading plot represents the metabolites responsible for separation on the score plots (Fig. 3B). HDG was located on the left of the score plot and most metabolites were located on the left of the loading plot. It indicated that the HDG contained relatively higher levels of most metabolites than the other cultivars. It is known that red rice seed contains relatively higher levels of phenolics and flavonoids than white rice seed $[24,30]$. However, Shen et al. [25] revealed that several white cultivars have a higher content of phenolic acids than red cultivars. In this study, relatively higher levels of caffeic acid, sinapinic acid, and vanillic acid were found in HDG, which has black-coloured hull, than in red cultivars. Phenolic acids are important building blocks employed in the biosynthesis of flavonoids. The level of raffinose was relatively higher in GR8 and HYJ (Additional file 1: Table S2). The content and composition of amino acids are shifted and raffinose is accumulated during seed development [31]. In this study, it was suggested that the expression pattern of genes or activity of enzymes associated with amino acid and raffinose metabolism might differ among the cultivars. No metabolite analysis data are available except for $\gamma$-oryzanols and octacosanol in the weedy rice, AM [32, 33]. The loading plot showed that AM contained relatively higher levels of leucine, isoleucine, methionine, mannitol, and phenylalanine than the other cultivars. Thus, chemometrics, combined with GC $\times$ GC-TOFMS, can facilitate a detailed metabolic profiling in rice for further studies in breeding programs.

\section{Supplementary Information}

The online version contains supplementary material available at https://doi. org/10.1186/s13765-021-00640-3.

Additional file 1: Table S1. Retention times (RT), relative retention times (RRT), and mass spectral data of polar metabolites as trimethylsilyl derivatives. Table S2. Composition and content (ratio/g) of polar metabolites in 8 rice cultivars on the $\mathrm{GC} \times \mathrm{GC}$-TOFMS.

\section{Acknowledgements}

This work was supported by Incheon National University Research Concentration Professors Grant in 2021

\section{Authors' contributions}

Conceptualization, methodology: JKK, SUP, SYK and S-AB. Data curation: SYK and S-AB. Formal analysis: YJP and SYK. Writing —original draft preparation: S-AB, SYK and JKK. Writing - review and editing: JKK, S-HL and TJK. Project administration: JKK and SUP. All authors read and approved the final manuscript.

\section{Declarations}

\section{Competing interests}

The authors declare that there is no competing interests.

\section{Author details}

${ }^{1}$ Division of Life Sciences and Bio-Resource and Environmental Center, College of Life Sciences and Bioengineering, Incheon National University, Incheon 22012, Republic of Korea. 'Division of Horticultural Biotechnology, School of Biotechnology, Hankyong National University, Anseong 17579, Republic of Korea. ${ }^{3}$ Department of Crop Science and Department of Smart Agriculture Systems, Chungnam National University, 99 Daehak-ro, Yuseong-gu, Daejeon 34134, Republic of Korea. ${ }^{4}$ Honam National Institute of Biological Resources, 58762 Mokpo, Republic of Korea. ${ }^{5}$ Nakdonggang National Institute of Biological Resources, 37242 Sangju, Republic of Korea.

Received: 13 June 2021 Accepted: 1 September 2021

Published online: 13 September 2021

\section{References}

1. Kim JK, Park SY, Lim SH, Yeo Y, Cho HS, Ha SH (2013) Comparative metabolic profiling of pigmented rice (Oryza sativa L.) cultivars reveals primary metabolites are correlated with secondary metabolites. J Cereal Sci 57:14-20. https://doi.org/10.1016/j.jcs.2012.09.012

2. Park SY, Lim SH, Ha SH, Yeo Y, Park WT, Kwon DY, Park SU, Kim JK (2013) Metabolite profiling approach reveals the interface of primary and secondary metabolism in colored cauliflowers (Brassica oleracea L. ssp. botrytis). J Agric Food Chem 61:6999-7007. https://doi.org/10.1021/jf401 330 e

3. Park CH, Yeo HJ, Park YE, Baek SA, Kim JK, Park SU (2019) Transcriptome analysis and metabolic profiling of Lycoris radiata. Biology 8:63. https:// doi.org/10.3390/biology8030063

4. Park Y, Baek SA, Choi Y, Kim JK, Park SU (2019) Metabolic profiling of nine Mentha species and prediction of their antioxidant properties using chemometrics. Molecules 24:258. https://doi.org/10.3390/molecules2 4020258

5. Bahaghigha DH, Freye CE, Synovec RE (2019) Recent advances in modulator technology for comprehensive two dimensional gas chromatography. Trac-Trend Anal Chem 113:379-391. https://doi.org/10.1016/j.trac.2018. 04.016

6. Winnike JH, Wei X, Knagge KJ, Colman SD, Gregory SG, Zhang X (2015) Comparison of GC-MS and GC $\times$ GC-MS in the analysis of human serum samples for biomarker discovery. J Proteome Res 14:1810-1817. https:// doi.org/10.1021/pr5011923 
7. Almstetter MF, Oefner PJ, Dettmer K (2012) Comprehensive twodimensional gas chromatography in metabolomics. Anal Bioanal Chem 402:1993-2013. https://doi.org/10.1007/s00216-011-5630-y

8. Prebihalo SE, Berrier KL, Freye CE, Bahaghighat HD, Moore NR, David PK, Synovec RE (2018) Multidimensional gas chromatography: advances in instrumentation, chemometrics, and applications. Anal Chem 90:505532. https://doi.org/10.1021/acs.analchem.7b04226

9. Pierce KM, Hoggard JC, Mohler RE, Synovec RE (2008) Recent advancements in comprehensive two-dimensional separations with chemometrics. J Chromatogr A 1184:341-352. https://doi.org/10.1016/j.chroma. 2007.07.059

10. Kusano M, Fukushima A, Kobayashi M, Hayashi N, Jonsson P, Moritz T, Ebana K, Saito K (2007) Application of a metabolomic method combining one-dimensional and two-dimensional gas chromatography-time-offlight/mass spectrometry to metabolic phenotyping of natural variants in rice. J Chromatogr B 855:71-79. https://doi.org/10.1016/j.jchromb.2007. 05.002

11. Allwood WJ, Erban A, de Koning S, Dunn WB, Luedemann A, Lommen A, Kay L, Löscher R, Kopka J, Goodacre R (2009) Inter-laboratory reproducibility of fast gas chromatography-electron impact-time of flight mass spectrometry (GC-EI-TOF/MS) based plant metabolomics. Metabolomics 5:479-496. https://doi.org/10.1007/s11306-009-0169-z

12. Xu B, Zhang L, Ma F, Zhang W, Wang X, Zhang Q, Luo D, Ma H, Li P (2018) Determination of free steroidal compounds in vegetable oils by comprehensive two-dimensional gas chromatography coupled to time-of-flight mass spectrometry. Food Chem 245:415-425. https://doi.org/10.1016/j. foodchem.2017.10.114

13. Pierce KM, Hope JL, Hoggard JC, Synovec RE (2006) A principal component analysis based method to discover chemical differences in comprehensive two-dimensional gas chromatography with time-of-flight mass spectrometry (GC $\times$ GC-TOFMS) separations of metabolites in plant samples. Talanta 70:797-804. https://doi.org/10.1016/j.talanta.2006.01. 038

14. Salvador ÂC, Baptista I, Barros AS, Gomes NC, Cunha Â, Almeida A, Rocha SM (2013) Can volatile organic metabolites be used to simultaneously assess microbial and mite contamination level in cereal grains and coffee beans? PLoS ONE 8:e59338. https://doi.org/10.1371/journal.pone.00593 38

15. Hurtado C, Parastar H, Matamoros V, Piña B, Tauler R, Bayona JM (2017) Linking the morphological and metabolomic response of Lactuca sativa $L$ exposed to emerging contaminants using GC $\times$ GC-MS and chemometric tools. Sci Rep 7:1-13. https://doi.org/10.1038/s41598-017-06773-0

16. Lim V, Gorji SG, Daygon VD, Melissa F (2020) Untargeted and targeted metabolomic profiling of Australian indigenous fruits. Metabolites 10:114. https://doi.org/10.3390/metabo10030114

17. Daygon VD, Calingacion M, Forster LC, De Voss JJ, Schwartz BD, Ovenden B, Alonso DE, McCouch SR, Garson MJ, Fitzgerald MA (2017) Metabolomics and genomics combine to unravel the pathway for the presence of fragrance in rice. Sci Rep 7:8767. https://doi.org/10.1038/ s41598-017-07693-9

18. Setyaningsih W, Majchrzak T, Dymerski T, Namieśnik J, Palma M (2019) Key-marker volatile compounds in aromatic rice (Oryza Sativa) grains: an HS-SPME extraction method combined with GC $\times$ GC-TOFMS. Molecules 24:4180. https://doi.org/10.3390/molecules24224180

19. Shen S, Weng R, Li L, Xu X, Bai Y, Liu H (2016) Metabolomic analysis of mouse embryonic fibroblast cells in response to autophagy induced by acute starvation. Sci Rep 6:34075. https://doi.org/10.1038/srep34075

20. Kim TJ, Hyeon HJ, Park NI, Yi TG, Lim SH, Park SY, Ha SH, Kim JK (2020) A high-throughput platform for interpretation of metabolite profile data from pepper (Capsicum) fruits of 13 phenotypes associated with different fruit maturity. Food Chem 331:127286. https://doi.org/10.1016/j.foodc hem.2020.127286

21. You MK, Lee YJ, Kim JK, Baek SA, Jeon YA, Lim SH, Ha SH (2020) The organ-specific differential roles of rice DXS and DXR, the first two enzymes of the MEP pathway, in carotenoid metabolism in Oryza sativa leaves and seeds. BMC Plant Biol 20:167. https://doi.org/10.1186/ s12870-020-02357-9

22. Deng Y, Lu S (2017) Biosynthesis and regulation of phenylpropanoids in plants. CRC Crit Rev Plant Sci 36:257-290. https://doi.org/10.1080/07352 689.2017.1402852

23. Kim JK, Lee SY, Chu SM, Lim SH, Suh SC, Lee YT, Cho HS, Ha SH (2010) Variation and correlation analysis of flavonoids and carotenoids in Korean pigmented rice (Oryza sativa L.) cultivars. J Agric Food Chem 58:1280412809. https://doi.org/10.1021/jf103277g

24. Jun HI, Song GS, Yang El, Youn Y, Kim YS (2012) Antioxidant activities and phenolic compounds of pigmented rice bran extracts. J Food Sci 77:C759-C764. https://doi.org/10.1111/j.1750-3841.2012.02763.x

25. Shen Y, Jin L, Xiao P, Lu Y, Bao J (2009) Total phenolics, flavonoids, antioxidant capacity in rice grain and their relations to grain color, size and weight. J Cereal Sci 49:106-111. https://doi.org/10.1016/j.jcs.2008.07.010

26. Food and drug administration (2018) Bioanalytical method validation guidance for industry. https://www.fda.gov/files/drugs/published/Bioan alytical-Method-Validation-Guidance-for-Industry.pdf. Accessed 13 Jan 2021

27. Welthagen W, Shellie RA, Spranger J, Ristow M, Zimmermann R, Fiehn O (2005) Comprehensive two-dimensional gas chromatography-time-offlight mass spectrometry (GC $\times$ GC-TOF) for high resolution metaboIomics: biomarker discovery on spleen tissue extracts of obese NZO compared to lean C57BL/6 mice. Metabolomics 1:65-73. https://doi.org/ 10.1007/s11306-005-1108-2

28. Ahn YG, Jeon SH, Lim HB, Choi NR, Hwang GS, Kim YP, Lee JY (2018) Analysis of polycyclic aromatic hydrocarbons in ambient aerosols by using one-dimensional and comprehensive two-dimensional gas chromatography combined with mass spectrometric method: a comparative study. J Anal Methods Chem. https://doi.org/10.1155/2018/8341630

29. Kim TJ, Park JG, Ahn SK, Kim KW, Choi J, Kim HY, Ha SH, Seo WD, Kim JK (2020) Discrimination of adzuki bean (Vigna angularis) geographical origin by targeted and non-targeted metabolite profiling with gas chromatography time-of-flight mass spectrometry. Metabolites 10:112. https://doi.org/10.3390/metabo10030112

30. Kim GR, Jung ES, Lee S, Lim SH, Ha SH, Lee CH (2014) Combined mass spectrometry-based metabolite profiling of different pigmented rice (Oryza sativa L.) seeds and correlation with antioxidant activities. Molecules 19:15679-15686. https://doi.org/10.3390/molecules191015673

31. Rosental L, Nonogaki H, Fait A (2014) Activation and regulation of primary metabolism during seed germination. Seed Sci Res 24:1-15. https://doi. org/10.1017/S0960258513000391

32. Lee MC, Lee DJ, Lee GA, Park HJ, Lee JR, Choi YM, Lee SK, Jung Y, Cho YG, Song JY (2013) Analysis and comparison of the $\gamma$-oryzanol content based on phylogenetic groups in Korean landraces of rice (Oryza sativa L.). Plant Breed Biotechnol 1:58-69. https://doi.org/10.9787/PBB.2013.1.1.058

33. Cho YH, Farhoudi R, Farooq M, Lee DJ (2017) Evaluating Korean rice genotypes and landraces for octacosanol contents and antioxidant activity. Nat Prod Res 31:2778-2782. https://doi.org/10.1080/14786419.2017. 1292271

\section{Publisher's Note}

Springer Nature remains neutral with regard to jurisdictional claims in published maps and institutional affiliations. 